\title{
SEMINORMALITY OF OPERATORS FROM THEIR TENSOR PRODUCT
}

\author{
JAN STOCHEL \\ (Communicated by Palle E. T. Jorgensen ) \\ Wtodzimierz Mlak in memoriam
}

\begin{abstract}
The question of seminormality of tensor products of nonzero bounded linear operators on Hilbert spaces is investigated. It is shown that $A \otimes B$ is subnormal if and only if so are $A$ and $B$.
\end{abstract}

\section{INTRODUCTION}

It is well known that some properties of Hilbert space operators are preserved by the operation of taking the two-fold tensor product. However, it may happen that $A \otimes B$ possesses a property $P$, though $A$ and $B$ do not (mainly because $A \otimes B=(c A) \otimes\left(c^{-1} B\right)$ for any nonzero $\left.c \in \mathbb{C}\right)$. The aim of this paper is to show that if $P$ is related to seminormality, then the tensor product $A \otimes B$ of nonzero bounded linear operators possesses the property $P$ if and only if so do $c A$ and $c^{-1} B$ for some nonzero $c \in \mathbb{C}$. Since the same can be done for operators whose adjoints have the property $P$, we can concentrate on a more narrow class of properties. Let us recall some of them (cf. $[2,3,7])$.

We say that a bounded linear operator $A$ on a complex Hilbert space is quasinormal (resp. subnormal, hyponormal) if $A A^{*} A=A^{*} A^{2}$ (resp. $A$ is a restriction of a bounded normal operator to its closed invariant subspace, $A A^{*} \leq A^{*} A$ ).

\section{Preliminaries}

A sequence $\left\{\alpha_{n}\right\}_{n=0}^{\infty}$ of real numbers is said to be a Stieltjes moment sequence if there exists a positive Borel measure $\mu$ on the closed half-line $[0,+\infty)$ such that

$$
\alpha_{n}=\int_{0}^{+\infty} t^{n} d \mu(t), \quad n \geq 0 .
$$

The measure $\mu$ is called a representing measure of $\left\{\alpha_{n}\right\}_{n=0}^{\infty}$.

Recall two basic properties of Stieltjes moment sequences (cf. [1] and [9]).

(S1) If $\left\{\alpha_{n}\right\}_{n=0}^{\infty}$ and $\left\{\beta_{n}\right\}_{n=0}^{\infty}$ are Stieltjes moment sequences, then so is their product $\left\{\alpha_{n} \beta_{n}\right\}_{n=0}^{\infty}$.

Received by the editors July 6, 1994 .

1991 Mathematics Subject Classification. Primary 47B20; Secondary 47A80.

Key words and phrases. Tensor product, seminormal operator, subnormal operator, Stieltjes moment sequence.

This work was supported by a grant of the Komitet Badań Naukowych, Warsaw. 
(S2) The class of all Stieltjes moment sequences is closed with respect to the topology of pointwise convergence.

The following two lemmas play the crucial role in this paper.

Lemma 1.1. If $\left\{\alpha_{n}\right\}_{n=0}^{\infty}$ is a Stieltjes moment sequence, then

(i) $\alpha_{n+1}^{2} \leq \alpha_{n} \alpha_{n+2}, n \geq 0$

(ii) $\alpha_{n}=0$ for every $n \geq 1$ if and only if there exists $k \geq 1$ such that $\alpha_{k}=0$.

Proof. Applying the Cauchy-Schwarz inequality we get (i). (ii) follows easily from the integral representation (1.1).

Lemma 1.2. Let $\left\{\alpha_{n}\right\}_{n=0}^{\infty}$ and $\left\{\beta_{n}\right\}_{n=0}^{\infty}$ be two sequences of positive real numbers. If $p=\lim \sup \alpha_{n+1} \alpha_{n}^{-1}<+\infty$ and $q=\lim \sup \beta_{n+1} \beta_{n}^{-1}<+\infty$, then the following conditions are equivalent:

(i) $\left\{\alpha_{n}\right\}_{n=0}^{\infty}$ and $\left\{\beta_{n}\right\}_{n=0}^{\infty}$ are Stieltjes moment sequences.

(ii) For every integer $s \geq 0,\left\{\alpha_{n} \beta_{n+s}\right\}_{n=0}^{\infty}$ and $\left\{\alpha_{n+s} \beta_{n}\right\}_{n=0}^{\infty}$ are Stieltjes moment sequences.

Proof. The implication (i) $\Rightarrow$ (ii) follows from (S1).

(ii) $\Rightarrow$ (i). Put $p_{n}=\alpha_{n+1} \alpha_{n}^{-1}$ and $q_{n}=\beta_{n+1} \beta_{n}^{-1}, n \geq 0$. Applying Lemma 1.1(i) to the Stieltjes moment sequence $\left\{\alpha_{n} \beta_{n+s}\right\}_{n=0}^{\infty}(s \geq 0)$, we get

$$
p_{n} q_{n+s} \leq p_{n+1} q_{n+s+1}, \quad n \geq 0, s \geq 0 .
$$

Thus the sequence $\left\{p_{n} q_{n}\right\}_{n=0}^{\infty}$ is weakly increasing, which in turn implies

$$
0<\lim _{n \rightarrow \infty} p_{n} q_{n} \leq \limsup _{n \rightarrow \infty} p_{n} \limsup _{n \rightarrow \infty} q_{n}=p q .
$$

This means that $0<p, q<+\infty$. It follows from (1.2) that

$$
p_{n}=q^{-1} \limsup _{s \rightarrow \infty} p_{n} q_{n+s} \leq q^{-1} \limsup _{s \rightarrow \infty} p_{n+1} q_{n+s+1}=p_{n+1}, \quad n \geq 0 .
$$

Since the sequence $\left\{p_{n}\right\}_{n=0}^{\infty}$ is weakly increasing, there exists $\lim _{n \rightarrow \infty} p_{n}=p$. Thus

$$
\lim _{s \rightarrow \infty} \frac{\alpha_{n+s}}{\alpha_{s}}=\lim _{s \rightarrow \infty}\left(p_{s} p_{s+1} \cdot \ldots \cdot p_{s+n-1}\right)=p^{n}, \quad n \geq 0,
$$

which gives us

$$
p^{n} \beta_{n}=\lim _{s \rightarrow \infty}\left(\frac{\alpha_{n+s}}{\alpha_{s}} \beta_{n}\right), \quad n \geq 0 .
$$

It follows from (S2) that $\left\{p^{n} \beta_{n}\right\}_{n=0}^{\infty}$ and consequently $\left\{\beta_{n}\right\}_{n=0}^{\infty}$ are Stieltjes moment sequences. Similar arguments apply to the sequence $\left\{\alpha_{n}\right\}_{n=0}^{\infty}$. This completes the proof.

\section{MAIN RESUlts}

From now on, $H$ and $K$ are fixed complex Hilbert spaces and $\mathcal{B}(H)$ stands for the $C^{*}$-algebra of all bounded linear operators on $H$.

Let us begin with proving two results that will be of use later. 
Proposition 2.1. If $A_{1}, A_{2} \in \mathcal{B}(H)$ and $B_{1}, B_{2} \in \mathcal{B}(K)$ are nonzero operators, then $A_{1} \otimes B_{1}=A_{2} \otimes B_{2}$ if and only if there exists $c \in \mathbb{C} \backslash\{0\}$ such that $A_{2}=c A_{1}$ and $B_{2}=c^{-1} B_{1}$.

Proof. First we show that if $f_{1} \otimes g_{1}=f_{2} \otimes g_{2} \neq 0$, then there exists $c \in \mathbb{C} \backslash\{0\}$ such that $f_{2}=c f_{1}$ and $g_{2}=c^{-1} g_{1}$. Indeed, due to our assumption $\left(f_{1}, \cdot\right) g_{1}=\left(f_{2}, \cdot\right) g_{2}$ and $\left(g_{1}, \cdot\right) f_{1}=\left(g_{2}, \cdot\right) f_{2}$ and consequently there are $c, d \neq 0$ such that $f_{2}=c f_{1}$ and $g_{2}=d g_{1}$. Substituting these vectors into the original equality we get $d=c^{-1}$.

Assume that $A_{1} f \otimes B_{1} g=A_{2} f \otimes B_{2} g$ for all $f \in H, g \in K$. Then one can show that $\operatorname{ker} A_{1}=\operatorname{ker} A_{2}$ and $\operatorname{ker} B_{1}=\operatorname{ker} B_{2}$. In virtue of the previous paragraph, we can attach to each pair of nonzero vectors $f \in H \ominus \operatorname{ker} A_{1}$ and $g \in K \ominus \operatorname{ker} B_{1}$ a unique nonzero complex scalar $c$ such that $A_{2} f=c A_{1} f$ and $B_{2} g=c^{-1} B_{1} g$. It is easily seen that $c$ does not depend on $f$ 's and $g$ 's, which completes the proof.

Proposition 2.2. Let $A_{1}, A_{2} \in \mathcal{B}(H)$ and $B_{1}, B_{2} \in \mathcal{B}(K)$ be nonnegative operators. If $A_{1} \neq 0$ and $B_{1} \neq 0$, then the following conditions are equivalent:

(i) $A_{1} \otimes B_{1} \leq A_{2} \otimes B_{2}$.

(ii) There exists $c>0$ such that $A_{1} \leq c A_{2}$ and $B_{1} \leq c^{-1} B_{2}$.

Proof. Denote by $C_{j}$ and $D_{j}$ the square roots of $A_{j}$ and $B_{j}$, respectively $(j=1,2)$.

(ii) $\Rightarrow$ (i). Since $\left\|C_{1} f\right\|^{2} \leq c\left\|C_{2} f\right\|^{2}$ for $f \in H$, there exists $S \in \mathcal{B}(H)$ such that $\|S\| \leq c^{1 / 2}$ and $C_{1}=S C_{2}$ (cf. [2], Lemma 1.1). Likewise there exists $T \in \mathcal{B}(K)$ such that $\|T\| \leq c^{-1 / 2}$ and $D_{1}=T D_{2}$. Consequently

$$
\begin{aligned}
& \left(A_{1} \otimes B_{1} h, h\right)=\left\|C_{1} \otimes D_{1} h\right\|^{2}=\left\|(S \otimes T)\left(C_{2} \otimes D_{2}\right) h\right\|^{2} \\
& \quad \leq\|S\|^{2}\|T\|^{2}\left\|\left(C_{2} \otimes D_{2}\right) h\right\|^{2} \leq\left(A_{2} \otimes B_{2} h, h\right), \quad h \in H \otimes K .
\end{aligned}
$$

(i) $\Rightarrow$ (ii). It follows from (i) that

$$
\left\|C_{1} f\right\|\left\|D_{1} g\right\| \leq\left\|C_{2} f\right\|\left\|D_{2} g\right\|, \quad f \in H, g \in K .
$$

First notice that $\operatorname{ker} C_{2} \subseteq \operatorname{ker} C_{1}$ and $\operatorname{ker} D_{2} \subseteq \operatorname{ker} D_{1}$. Indeed, if $C_{2} f=0$, then by (2.1) we have $\left\|C_{1} f\right\|\left\|D_{1} g\right\|=0$ for every $g \in K$. However $D_{1} \neq 0$, so $C_{1} f=0$ (similarly we show the other inclusion). The above remark and (2.1) imply that there exists $0<c<+\infty$ such that

$$
\sup _{C_{1} f \neq 0}\left\|C_{1} f\right\|\left\|C_{2} f\right\|^{-1} \leq c^{1 / 2} \leq \inf _{D_{1} g \neq 0}\left\|D_{2} g\right\|\left\|D_{1} g\right\|^{-1} .
$$

Hence

$$
\left(A_{1} f, f\right)=\left\|C_{1} f\right\|^{2} \leq c\left\|C_{2} f\right\|^{2}=c\left(A_{2} f, f\right), \quad f \in H .
$$

Similarly we show that $\left(B_{1} g, g\right) \leq c^{-1}\left(B_{2} g, g\right)$ for $g \in K$, which completes the proof.

In the proof of the main result of this paper we will make use of the following version of Lambert's criterion for subnormality of bounded operators.

Proposition 2.3. If $A \in \mathcal{B}(H)$, then the following conditions are equivalent:

(i) $A$ is subnormal.

(ii) For every $f \in H,\left\{\left\|A^{n} f\right\|^{2}\right\}_{n=0}^{\infty}$ is a Stieltjes moment sequence.

(iii) For every $f \in H \ominus \operatorname{ker} A,\left\{\left\|A^{n} f\right\|^{2}\right\}_{n=0}^{\infty}$ is a Stieltjes moment sequence. 
Proof. The equivalence (i) $\Leftrightarrow$ (ii) is essentially due to Lambert (cf. $[6,10])$.

(iii) $\Rightarrow$ (ii). If $f \in H$, then $f=g \oplus h$, where $g \in \operatorname{ker} A, h \in H \ominus \operatorname{ker} A$. Therefore $\left\|A^{n} f\right\|^{2}=\int_{0}^{\infty} t^{n} d\left(\mu+\|g\|^{2} \delta_{0}\right)(t)$ for $n \geq 0$, where $\mu$ is a representing measure of $\left\{\left\|A^{n} h\right\|^{2}\right\}_{n=0}^{\infty}$ and $\delta_{0}$ is the probability measure concentrated on $\{0\}$. This completes the proof.

We are now in a position to prove the main result of the paper.

Theorem 2.4. If $A \in \mathcal{B}(H)$ and $B \in \mathcal{B}(K)$ are nonzero operators, then

(i) $A \otimes B$ is isometric (resp. unitary, selfadjoint, nonnegative) if and only if so are $c A$ and $c^{-1} B$ for some $c \in \mathbb{C} \backslash\{0\}$,

(ii) $A \otimes B$ is normal (resp. quasinormal, subnormal, hyponormal) if and only if so are $A$ and $B$.

Proof. (i) Among the properties mentioned in (i), only the positivity needs some explanation, as the other ones can be easily deduced from Proposition 2.1 (see also Normality below).

Positivity. If $A \geq 0$ and $B \geq 0$, then $A \otimes B=\left(A^{1 / 2} \otimes B^{1 / 2}\right)^{2} \geq 0$. Conversely, if $A \otimes B \geq 0$, then by (i) there exists $c \neq 0$ such that $c A$ and $c^{-1} B$ are selfadjoint. Therefore we can assume without loss of generality that $A$ and $B$ are selfadjoint and

$$
(A f, f)(B g, g) \geq 0, \quad f \in H, g \in K \text {. }
$$

Since $A \neq 0$, there exists $f_{0}$ such that $\left(A f_{0}, f_{0}\right) \in \mathbb{R} \backslash\{0\}$. If $\left(A f_{0}, f_{0}\right)>0$, then by (2.2) we have $B \geq 0$. However, $B \neq 0$, so there exists $g_{0}$ such that $\left(B g_{0}, g_{0}\right)>0$. Applying once more $(2.2)$ we obtain $A \geq 0$. The other case $\left(A f_{0}, f_{0}\right)<0$ leads to $-A \geq 0$ and $-B \geq 0$.

(ii) Normality. If $A \otimes B$ is normal, then by Proposition 2.1 there exists $c \neq 0$ such that $A^{*} A=c A A^{*}$ and $B^{*} B=c^{-1} B B^{*}$. Consequently $c>0,\|A\|^{2}=c\|A\|^{2}$ and $\|B\|^{2}=c^{-1}\|B\|^{2}$, which is possible only if $c=1$. Thus $A$ and $B$ are normal. The converse implication is obvious.

Quasinormality. If $A \otimes B$ is quasinormal, then by Proposition 2.1 there exists $c \neq 0$ such that $A A^{*} A=c A^{*} A^{2}$ and $B B^{*} B=c^{-1} B^{*} B^{2}$. This in turn implies that $\left(A^{*} A\right)^{2}=c\left(A^{2}\right)^{*} A^{2}$ and $\left(B^{*} B\right)^{2}=c^{-1}\left(B^{2}\right)^{*} B^{2}$. Consequently $c>0,\|A\|^{4}=$ $c\left\|A^{2}\right\|^{2} \leq c\|A\|^{4}$ and $\|B\|^{4}=c^{-1}\left\|B^{2}\right\|^{2} \leq c^{-1}\|B\|^{4}$, which yields $c=1$. Therefore $A$ and $B$ are quasinormal. The converse implication is obvious.

Subnormality. Assume that $A \otimes B$ is subnormal. Put $\alpha_{n}(f):=\left\|A^{n} f\right\|^{2}$ and $\beta_{n}(g):=\left\|B^{n} g\right\|^{2}$ for $f \in H, g \in K$ and $n \geq 0$. Then $\alpha_{n+i}(f) \beta_{n+j}(g)=$ $\left\|(A \otimes B)^{n}\left(A^{i} f \otimes B^{j} g\right)\right\|^{2}$ for $i, j \geq 0$, so by Proposition 2.3 we have

$$
\begin{aligned}
\left\{\alpha_{n+i}(f) \beta_{n+j}(g)\right\}_{n=0}^{\infty} & \text { is a Stieltjes moment sequence } \\
& \text { for all } f \in H, g \in K \text { and } i, j \geq 0 .
\end{aligned}
$$

Note that if $f \notin \operatorname{ker} A$ and $g \notin \operatorname{ker} B$, then $\alpha_{n}(f)>0$ and $\beta_{n}(g)>0$ for every $n \geq 0$. Indeed, if $\alpha_{k}(f)=0$ for some $k \geq 1\left(\alpha_{0}(f) \neq 0\right.$ !), then by $B \neq 0$ there exists $h \notin \operatorname{ker} B$. It follows from (2.3) and Lemma 1.1(ii) that $\|A f\|^{2}\|B h\|^{2}=$ $\alpha_{1}(f) \beta_{1}(h)=0$, which contradicts $A f \neq 0$ and $B h \neq 0$. Similar arguments apply to $\left\{\beta_{n}(g)\right\}_{n=0}^{\infty}$. 
Take arbitrary nonzero vectors $f \in H \ominus \operatorname{ker} A$ and $g \in K \ominus \operatorname{ker} B$. Then $\lim \sup _{n \rightarrow \infty} \alpha_{n+1}(f) \alpha_{n}^{-1}(f) \leq\|A\|^{2}<+\infty$ and $\limsup _{n \rightarrow \infty} \beta_{n+1}(g) \beta_{n}^{-1}(g) \leq$ $\|B\|^{2}<+\infty$. This and condition (2.3) imply-via Lemma 1.2 - that $\left\{\alpha_{n}(f)\right\}_{n=0}^{\infty}$ and $\left\{\beta_{n}(g)\right\}_{n=0}^{\infty}$ are Stieltjes moment sequences. Hence, by Proposition 2.3, $A$ and $B$ are subnormal. The converse implication is obvious.

Hyponormality. The sufficiency can be directly derived from Proposition 2.2. If $A \otimes B$ is hyponormal, then $\left(A A^{*} \otimes B B^{*}\right) \leq A^{*} A \otimes B^{*} B$ and consequently-by Proposition 2.2-there exists $c>0$ such that $A A^{*} \leq c A^{*} A$ and $B B^{*} \leq c^{-1} B^{*} B$. Hence $\|A\|^{2} \leq c\|A\|^{2}$ and $\|B\|^{2} \leq c^{-1}\|B\|^{2}$. Since both $A$ and $B$ are nonzero, we come to the conclusion that $c=1$. This means that $A$ and $B$ are hyponormal.

\section{COMments AND CONCLUding REMARKS}

The following example shows that the condition (ii) of Lemma 1.2 cannot be weakened.

Example 3.1. For every integer $s \geq 1$, there exist two sequences $\left\{\alpha_{n}\right\}_{n=0}^{\infty}$ and $\left\{\beta_{n}\right\}_{n=0}^{\infty}$ of positive real numbers such that

(i) $\lim \sup \alpha_{n+1} \alpha_{n}^{-1}<+\infty$ and $\lim \sup \beta_{n+1} \beta_{n}^{-1}<+\infty$,

(ii) $\left\{\alpha_{n}\right\}_{n=0}^{\infty}$ is not a Stieltjes moment sequence,

(iii) for every $j=0,1, \ldots, s,\left\{\alpha_{n} \beta_{n+j}\right\}_{n=0}^{\infty}$ and $\left\{\alpha_{n+j} \beta_{n}\right\}_{n=0}^{\infty}$ are Stieltjes moment sequences.

Take an arbitrary Stieltjes moment sequence $\left\{p_{n}\right\}_{n=0}^{\infty}$ of positive real numbers. Choose $\alpha_{0}$ and $\beta_{0}$ so that $\alpha_{0} \beta_{0}=p_{0} \cdots p_{s-1}$. Put $\alpha_{n}=\beta_{0}^{-1} p_{0} \cdots p_{n+s-1}$ and $\beta_{n}=$ $\beta_{0}\left(p_{0} \cdots p_{n-1}\right)^{-1}$ for $n \geq 1$. Then $\left\{\alpha_{n} \beta_{n+j}\right\}_{n=0}^{\infty}$ is the constant sequence for $j=s$ and the finite product $\prod_{k=j}^{s-1} p_{n+k}$ of Stieltjes moment sequences for $j<s$. It follows from (S1) that $\left\{\alpha_{n} \beta_{n+j}\right\}_{n=0}^{\infty}, j=0,1, \ldots, s$, are Stieltjes moment sequences. The same reasoning applies to the sequences $\left\{\alpha_{n+j} \beta_{n}\right\}_{n=0}^{\infty}, j=0,1, \ldots, s$. If additionally there exist $\delta>0$ and $M>0$ such that $\delta \leq p_{n} \leq M$ for all $n \geq 0$ (or equivalently $\left\{p_{n}\right\}_{n=0}^{\infty}$ is a Hausdorff moment sequence whose representing measure has 1 as an atom), then (i) holds. To get (ii) it is sufficient to assume that $\left\{p_{n}\right\}_{n=0}^{\infty}$ is strictly decreasing. Indeed, if $\left\{\alpha_{n}\right\}_{n=0}^{\infty}$ were a Stieltjes moment sequence, the sequence $\left\{\alpha_{n+1} \alpha_{n}{ }^{-1}\right\}_{n=0}^{\infty}$ would be weakly increasing (apply Lemma 1.1(i)), which would contradict the equality $\alpha_{n+1} \alpha_{n}{ }^{-1}=p_{n+s}, n \geq 0$. The sequence $p_{n}:=1+(n+1)^{-1}$ meets all the requirements.

A part of Theorem 2.4(ii) concerning subnormality of $A \otimes B$ can be directly inferred from the definition of subnormality, provided $A$ and $B$ have nonempty point spectrum. Among operators with empty point spectrum there are injective weighted shifts. This particular case is precisely covered by Lemma 1.2. Indeed, since $A \otimes B$ is unitarily equivalent to $\left(\sum_{k=0}^{\infty} \oplus A_{k}\right) \oplus\left(\sum_{k=1}^{\infty} \oplus B_{k}\right)$, where $A$, $B, A_{k}$ and $B_{k}$ are weighted shifts with weights $\left\{\lambda_{n}\right\}_{n=0}^{\infty},\left\{\mu_{n}\right\}_{n=0}^{\infty},\left\{\lambda_{n+k} \mu_{n}\right\}_{n=0}^{\infty}$ and $\left\{\lambda_{n} \mu_{n+k}\right\}_{n=0}^{\infty}$, respectively, we can apply the Berger-Gellar-Wallen criterion for subnormality of weighted shifts (cf. $[4,5,8]$ ).

We conclude the paper with the following

Open Question. Is it true that if the two-fold symmetric tensor product of a nonzero bounded linear operator $A$ by itself is subnormal, then so is $A$ ?

We know the affirmative answer for injective weighted shifts. Indeed, in this particular case the symmetric tensor product of $A$ by itself is unitarily equivalent 
to the orthogonal sum $\sum_{k=0}^{\infty} \bigoplus S_{k}$ of weighted shifts $S_{k}$ with weights $\left\{\lambda_{n} \lambda_{n+k}\right\}_{n=0}^{\infty}$, $k \geq 0$, where $\left\{\lambda_{n}\right\}_{n=0}^{\infty}$ are weights of $A$. Applying the Berger-Gellar-Wallen theorem and Lemma 1.2 we get the conclusion.

\section{REFERENCES}

1. Ch. Berg, J. P. R. Christensen, and P. Ressel, Harmonic analysis on semigroups, Springer, Berlin, 1984. MR 86b:43001

2. K. Clancey, Seminormal operators, Lecture Notes in Math., vol. 742, Springer-Verlag, Berlin, Heidelberg, and New York, 1979, pp. 1-124. MR 81c:47002

3. J. B. Conway, The theory of subnormal operators, Math. Surveys Monographs, vol. 36, Amer. Math. Soc., Providence, RI, 1991. MR 92h:47026

4. R. Gellar and L. J. Wallen, Subnormal weighted shifts and the Halmos-Bram criterion, Proc. Japan. Acad. 46 (1970), 375-378. MR 51:8879

5. P. R. Halmos, Ten problems in Hilbert space, Bull. Amer. Math. Soc. 76 (1970), 887-933. MR 42:5066

6. A. Lambert, Subnormality and weighted shifts, J. London Math. Soc. 14 (1976), 476-480. MR 55:8866

7. M. Martin and M. Putinar, Lectures on hyponormal operators, Birkhäuser-Verlag, 1989. MR 91c: 47041

8. A. L. Shields, Weighted shift operators and analytic function theory, Math. Surveys Monographs, vol. 13, Amer. Math. Soc., Providence, RI, 1974, pp. 49-128. MR 50:14341

9. J. A. Shohat and J. D. Tamarkin, The problem of moments, Math. Surveys Monographs, vol. 1, Amer. Math. Soc., Providence, RI, 1943. MR 5:5c

10. J. Stochel and F. H. Szafraniec, On normal extensions of unbounded operators. II, Acta. Sci. Math. (Szeged) 53 (1989), 153-177. MR 91i:47032

Instytut Matematyki, Uniwersytet Jagielloński, ul. Reymonta 4, PL-30059 KraKÓw, POLAND

E-mail address: stochel@im.uj.edu.pl 